\title{
Erratum: "Ferroelectric Materials for Microwave Tunable Applications" [J. Electroceram. 11, 5 (2003)]
}

\author{
A.K. TAGANTSEV, V.O. SHERMAN, K.F. ASTAFIEV, J. VENKATESH \& N. SETTER \\ Ceramics Laboratory, Swiss Federal Institute of Technology, EPFL, 1015 Lausanne, Switzerland
}

Submitted November 16, 2004; Revised November 16, 2004; Accepted November 17, 2004

1. Equation (3.13) should read

$$
E_{N}=\frac{2}{\sqrt{27 \beta \varepsilon_{00}^{3} \varepsilon_{0}^{3}}}
$$

instead of

$$
E_{N}=\frac{2}{\sqrt{27 \beta \varepsilon_{00}^{3}}} .
$$

2. Equation (3.22) should read:

$$
\varepsilon_{\text {mix }}^{-1}\left(q, E_{0}\right)=\varepsilon_{0} \alpha^{*}+3 \beta \varepsilon_{0} P_{f}^{2}+\frac{q^{2}\left(\varepsilon_{b}-\varepsilon_{d}\right)}{\varepsilon_{d}\left[\varepsilon_{b} q+\varepsilon_{d}(1-q)\right]}-q \varepsilon_{0}\left(\alpha+3 \beta P_{f}^{2}\right)
$$

instead of:

$$
\varepsilon_{\text {mix }}^{-1}\left(q, E_{0}\right)=\varepsilon_{0} \alpha^{*}+3 \beta \varepsilon_{0} P_{f}^{2}-\frac{q^{2}\left(\varepsilon_{b}-\varepsilon_{d}\right)}{\varepsilon_{d}\left[\varepsilon_{b} q+\varepsilon_{d}(1-q)\right]}-q \varepsilon_{0}\left(\alpha+3 \beta P_{f}^{2}\right) .
$$

3. Sentence after Eq. (3.22) on page \#16 should read:

"In the limit of small concentration of the dielectric material in the mixture $(q \ll 1)$ and when $\varepsilon_{f} / \varepsilon_{d} \gg 1$, the last two terms in the Eq. (3.22) can be neglected and the renormalized electric field $E^{*}$ in Eq. (3.20) become equal to the electric field $E_{0}$."

instead of:

"In the limit of small concentration of the dielectric material in the mixture $(q \ll 1)$ and when $\varepsilon_{f} / \varepsilon_{d} \gg 1$, the last three terms in the Eq. (3.22) can be neglected and the renormalized electric field $E^{*}$ in Eq. (3.20) become equal to the electric field $E_{0}$."

4. Equation for the matrix $G_{t s}$ on page \#16 should read $G_{t s}=3 n_{t} n_{s}-\delta_{t s}$ instead of $G_{t s}=3 n+n s-\delta_{t s}$.

5. Sentence after equation for the matrix $G_{t s}$ on page \#16 should read:

"where $\vec{n}=\left(\vec{r}-\vec{r}_{i}\right) /\left|\vec{r}-\vec{r}_{i}\right|$ and $\delta_{t s}$ is the Kroneker symbol."

instead of:

"where $\vec{n}=\left(\vec{r}-\vec{r}_{i}\right) /\left|\vec{r}-\vec{r}_{i}\right|$ and $\delta$ is the Kroneker symbol. Where $\varphi$ and $\theta$ are the azimuthal and polar angles of the vector $\vec{r}-\vec{r}_{i}$." 
6. Sentence after Eq. (3.25a) on page \#16 should read:

"In this solution, when calculating the dielectric nonlinearity of the composite the second term in Eq. (3.25a) is neglected so that in the ferroelectric matrix one set $\vec{E}_{f} \approx \vec{E}$."

instead of:

"In this solution, when calculating the dielectric nonlinearity of the composite the second term in Eq. (3.27) is neglected so that in the ferroelectric matrix one set $\vec{E}_{f} \approx \vec{E}$."

7. Sentence after Eq. (3.26) on page \#17 should read:

"where $n_{r}$ is the tunability of the ferroelectric at the same value of the applied field."

instead of:

"where $n_{r, \text { mix }}$ is the tunability of the ferroelectric at the same value of the applied field."

8. The calculated value for $\mathrm{Ba}_{0.6} \mathrm{Sr}_{0.4} \mathrm{TiO}_{3}$ in Table 4 should read 0.1 instead of 0.7-1.4.

9. Figure 13 should read:
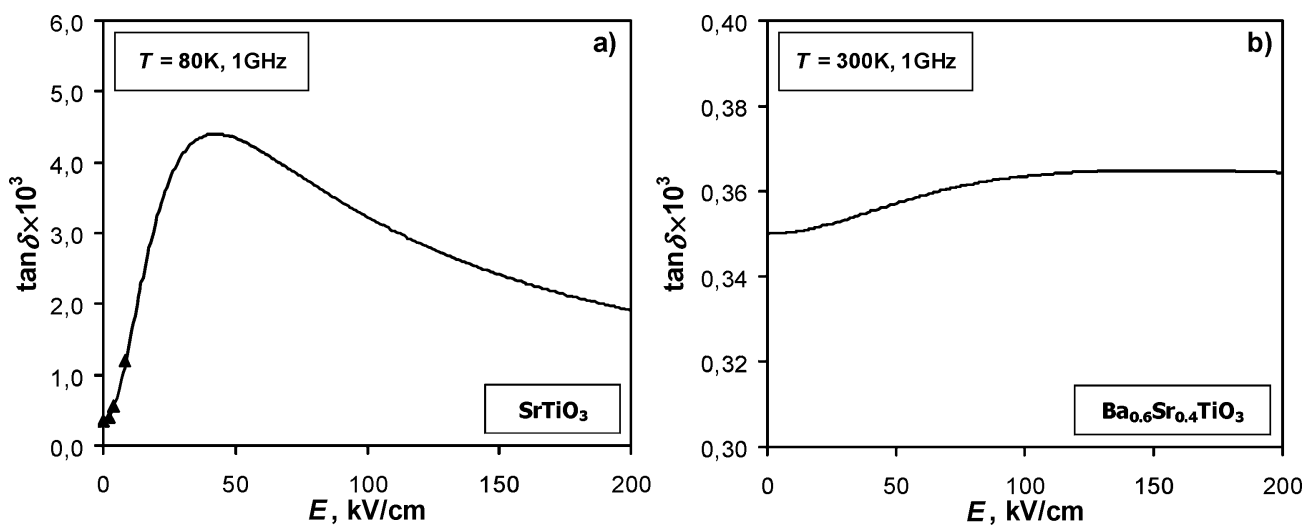

Fig. 13. Simulated field dependence of the loss tangent (solid lines) plotted together with experimental data [2] (black squares) for $\mathrm{SrTiO}_{3}(\mathrm{a})$ and $\mathrm{Ba}_{0.6} \mathrm{Sr}_{0.4} \mathrm{TiO}_{3}$ (b) crystals; $A_{\mathrm{SrTiO}_{3}}=17 \times 10^{-3} \mathrm{GHz}^{-1}, A_{\mathrm{Ba}_{0} 6 \mathrm{Sr}_{0.4} \mathrm{TiO}_{3}}=0.1 \times 10^{-3} \mathrm{GHz}^{-1}$. The values of tan $\delta(0)$ are taken from Refs. [2] and [68], respectively.

10. The dimension of the $\beta$ coefficient in Table 5 should read $\mathrm{JC}^{-4} \mathrm{~m}^{5}$ instead of $\mathrm{JC}^{-4} \mathrm{~m}^{-5}$.

11. Figure 15 should read:
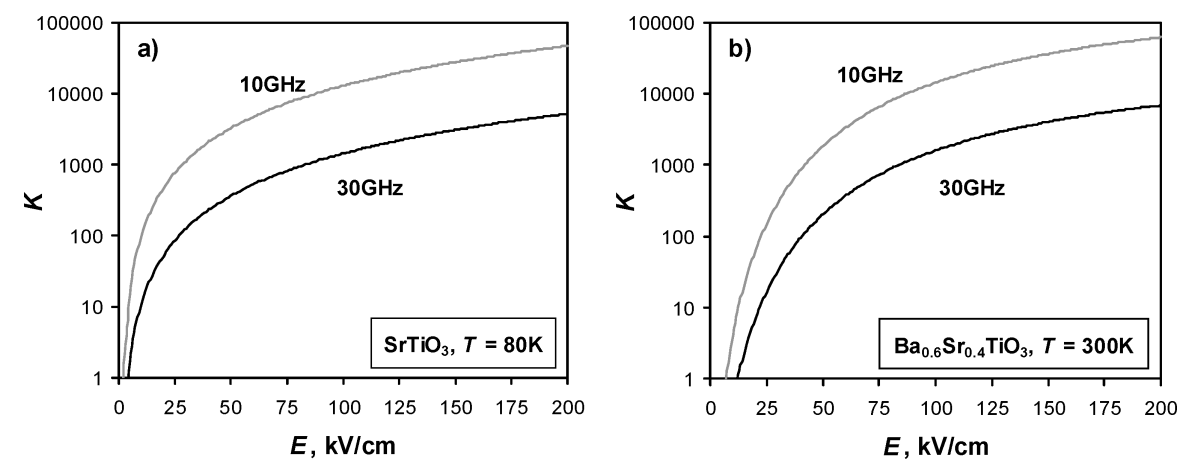

Fig. 15. Calculated field dependences of the quality factor $K$ for $\mathrm{SrTiO}_{3}$ (a) and $\mathrm{Ba}_{0.6} \mathrm{Sr}_{0.4} \mathrm{TiO}_{3}$ (b) ferroelectrics at frequencies of $10 \mathrm{GHz}$ (grey lines) and $30 \mathrm{GHz}$ (black lines). The calculations were performed for the same material parameters as those used for plotting the graphs in Fig. 13 [67]. 
12. Equation (3.59) should read:

$$
E_{e x t}=\alpha P-k \frac{\partial^{2} P}{\partial x^{2}}+\frac{1}{\varepsilon_{0} \varepsilon_{b}}(P-\bar{P})
$$

instead of:

$$
E_{e x t}=\alpha P-k \frac{\partial^{2} P}{\partial x^{2}}+\frac{1}{\varepsilon_{0}}(P-\bar{P})
$$

13. Equation (3.60) should read:

$$
P(x)=\frac{E_{e x t}}{\alpha}\left(1-\frac{\cosh \frac{x-h / 2}{\xi_{1}}}{\cosh \frac{h}{2 \xi_{1}}}\right) \frac{1}{1+2 \frac{\varepsilon}{\varepsilon_{b}} \frac{\xi_{1}}{h} \tanh \frac{h}{2 \xi_{1}}}
$$

instead of:

$$
P(x)=\frac{E_{e x t}}{\alpha}\left(1-\frac{\cosh \frac{x-h / 2}{\xi_{1}}}{\cosh \frac{h}{2 \xi_{1}}}\right) \frac{1}{1+2 \varepsilon \frac{\xi_{1}}{h} \tanh \frac{h}{2 \xi_{1}}} .
$$

14. Equation (3.61) should read:

$$
\varepsilon_{e f f}^{-1}=\varepsilon^{-1}+\frac{2 \xi_{1}}{h} \varepsilon_{b}^{-1}
$$

instead of:

$$
\varepsilon_{\text {eff }}^{-1}=\varepsilon^{-1}+\frac{2 \xi_{1}}{h}
$$

15. In the section (i) on page \#30, the sentence should read:

"However, this result may be taken only as qualitative since the theoretical situation corresponds to the limit of the range of applicability of the continuous Landau theory; the latter is applicable if the typical scale of the polarization variation ( $\xi$ in this case) is essentially larger than the lattice constant of the material (4 $\AA$ in the case of $\left.(\mathrm{Ba}, \mathrm{Sr}) \mathrm{TiO}_{3}\right)$."

instead of:

"However, this result may be taken only as quantitative since the theoretical situation corresponds to the limit of the range of applicability of the continuous Landau theory; the latter is applicable of the typical scale of the polarization variation ( $\xi$ in this case) is essentially larger than the lattice constant of the material ( $4 \AA$ in the case of $\left.(\mathrm{Ba}, \mathrm{Sr}) \mathrm{TiO}_{3}\right)$.".

16. In the section (ii) on page \#30, the sentence should read:

"According to Eq. (3.61) the expected relative correction to the bulk permittivity is about $2 \xi_{1} \varepsilon / h \varepsilon_{b}$, that is some $\sqrt{\varepsilon / \varepsilon_{b}}$ times stronger than in the case of the in-plane component."

instead of:

"According to Eq. (3.61) the expected relative correction to the bulk permittivity is about $2 \xi_{1} \varepsilon / h$, that is some $\sqrt{\varepsilon / \varepsilon_{b}}$ times stronger than in the case of the in-plane component." 
17. In the section (ii) on page \#30, the sentences should read:

"In this context, Eq. (3.61) might be used as a semi-empirical relation, $\xi_{1} / \varepsilon_{b}$ being a fitting parameter. An analysis of the thickness dependence of the out-of-plane component of dielectric constant in terms of Eq. (3.61) performed by Vendik and Zubko [82] for $(\mathrm{Ba}, \mathrm{Sr}) \mathrm{TiO}_{3}$ thin films yields the values of $\xi_{1} / \varepsilon_{b}$ in the range of $0.2-2.5 \AA . "$

instead of:

"In this context, Eq. (3.61) might be used as a semi-empirical relation $\xi_{1}$ being a fitting parameter. An analysis of the thickness dependence of the in-plane component of dielectric constant in terms of Eq. (3.61) performed by Vendik and Zubko [82] for $(\mathrm{Ba}, \mathrm{Sr}) \mathrm{TiO}_{3}$ thin films yields the values of $\xi_{1}$ in the range of 0.2-2.5 $\AA$."

18. Sentence before Eq. (3.71) on page \#32 should read: "In the case of the partial and full depletion, one finds" instead of: "In the case of the full and partial depletion, one finds".

19. Equation (3.71) should read:

$$
\frac{1}{\varepsilon_{\text {eff }}}=\frac{1}{\varepsilon}+\frac{2 \varepsilon_{0} \beta \rho_{0}^{2} W^{3}}{h}
$$

instead of:

$$
\frac{1}{\varepsilon_{\text {eff }}}=\frac{1}{\varepsilon}+\frac{4 \varepsilon_{0} \beta \rho_{0}^{2} W^{3}}{h} .
$$

20. Equation (3.72) should read:

$$
\frac{1}{\varepsilon_{\text {eff }}}=\frac{1}{\varepsilon}+\frac{\varepsilon_{0} \beta \rho_{0}^{2} h^{2}}{4}
$$

instead of:

$$
\frac{1}{\varepsilon_{\text {eff }}}=\frac{1}{\varepsilon}+\frac{\varepsilon_{0} \beta \rho_{0}^{2} h^{2}}{2} .
$$

21. The thickness of a dielectric layer in Table 6 on page \#32 for the case of full surface blocking of polarization should read $\xi_{1}=\xi / \sqrt{\varepsilon \varepsilon_{b}}$ instead of $\xi_{1}=\xi \sqrt{\varepsilon_{b} / \varepsilon}$.

22. Sentence on page $\# 32$ should read:

"For values of $W$ and $\rho_{0}$ compatible with the data on ferroelectric perovskite with metallic electrodes, $W=$ $0.2 \mu \mathrm{m}$ and $\rho_{0} \cong 1.6 \times 10^{-19} \times 10^{18} \mathrm{Ccm}^{-3}=0.16 \mathrm{Ccm}^{-3}[72,89,91]$ one finds that, in the case of partial depletion (where $h>2 W$ ), a single depletion layer works as a dielectric layer with $\varepsilon_{d}=1$ and thickness $h_{d}=\varepsilon_{0} \beta \rho_{0}^{2} W^{3} \approx 0.1 \AA . "$

instead of:

"For values of $W$ and $\rho_{0}$ compatible with the data on ferroelectric perovskite with metallic electrodes, $W=$ $0.2 \mu \mathrm{m}$ and $\rho_{0} \cong 1.6 \times 10^{-19} \times 10^{18} \mathrm{Ccm}^{-3}=0.16 \mathrm{Ccm}^{-3}[72,89,91]$ one finds that, in the case of partial depletion (where $h>2 W$ ), a single depletion layer works as a dielectric layer with $\varepsilon_{d}=1$ and thickness $h_{d}=2 \varepsilon_{0} \beta \rho_{0}^{2} W^{3} \approx 0.2 \AA . "$

23. The limit " $0.05<C[\mathrm{pF}]<1$ " for the capacitance $C$ in Table 7 for the resonance methods should be removed. 
24. The reference [33] should read:

"J.G. Colom, R.A. Rodrigues-Solis, J. Almodovar, and M. Castaneda, Integrated Ferroelectrics, 42, 313 (2002)." instead of:

"J.G. Colom, R.A. Rodrigues-Solis, J. Almodovar, and M. Castaneda, Integrated Ferroelectrics, 42, 313 (2001)." 25. The reference [68] should read:

“L.C. Sengupta, S. Stowell, E. Ngo, M.E. O’Day, and R. Lancto, Integrated Ferroelectrics, 8, 77 (1995).” instead of:

"L.C. Sengupta, S. Stowell, E. Ngo, M.E. O’Day, and R. Lancto, Integrated Ferroelectrics, 8, 821 (1995)." 26. The reference [137] should read:

"L.C. Sengupta and S. Sengupta, Mat. Res. Innovat., 2, 278 (1999)." instead of:

"L.C. Sengupta and S. Sengupta, Mat. Res. Innovat., 278 (1999).” 